\title{
Enhancement of the longitudinal transport by a weakly transversal drive
}

\author{
Ya-feng $\mathrm{He}^{1,2 *}$ and Bao-quan $\mathrm{Ai}^{1}$ \\ ${ }^{1}$ Laboratory of Quantum Information Technology, ICMP and SPTE, \\ South China Normal University, 510006 Guangzhou, China. \\ ${ }^{2}$ College of Physics Science and Technology, \\ Hebei University, 071002 Baoding, China
}

(Dated: November 19, 2018)

\begin{abstract}
Transport of Brownian particles in a two-dimensional asymmetric tube is investigated by applying a polarized field. From the Brownian dynamics simulations we find that the longitudinal current can be enhanced remarkably by applying a weakly transversal drive. Multiple current reversals can be realized by altering the driving frequency of the polarized field. By coupling the longitudinal and transversal forces together, one can control the particle transport flexibly.

PACS numbers: 05. 40. -a, 05. 60. -k, 07. 20. Pe
\end{abstract}

*Email: heyf@hbu.edu.cn 


\section{INTRODUCTION}

The problem of non-equilibrium-induced transport processes has attracted much interest in theoretical as well as experimental physics [1-4]. This subject was motivated by the challenge to explain unidirectional transport in biological systems [1], as well as their potential technological applications ranging from classical non-equilibrium models [4, 5] to quantum systems [6, 7]. In these systems directed-Brownian-motion of particles is generated by non-equilibrium noise in the absence of any net macroscopic forces and potential gradients. Ratchets [8-11] have been proposed to model the unidirectional motion due to the zero-mean non-equilibrium fluctuation.

Recently, ratchet effects were realized in many experimental works [12 15, 17, 18]. Savel'ev and Nori [12] proposed devices for controlling the motion of flux quanta in superconductors and could address a central problem in many superconducting devices. They also demonstrated experimentally how to guide flux quanta in layered superconductors by using a drive that is asymmetric in time instead of being asymmetric in space \13]. Once or multiple reversal in the direction of the vortex flow has been observed [14, 15]. Motivated by the theoretical study in Ref. [16], Kalman and co-workers [17] studied ion current rectification in single conical nanopores in polymer films in the presence of two rectangular voltage signals. In addition, the ratchet effects were also realized in cold atoms. Renzoni and co-workers [18] demonstrate experimentally a gating ratchet with cold rubidium atoms in a driven near-resonant optical lattice. A single harmonic periodic modulation of the potential depth is applied, together with a single-harmonic rocking force. Directed motion is observed as a result of the breaking of the symmetries of the system.

Most studies have revolved around the energy barriers [19, 20]. However, in many transport phenomena [21], such as those taking place in micro- and nano-pores, zeolites, biological cells, ion channels, nanoporous materials and microfuidic devices etched with grooves and chambers, Brownian particles, instead of diffusing freely in the host liquid phase, undergo a constrained motion. Recently, Reguera and coworkers [22] used the mesoscopic nonequilibrium thermodynamics theory to derive the general kinetic equation of the system and studied the current and the diffusion of Brownian particles moving in a symmetric channel with a biased external force. Ai and co-workers [23] studied the transport driven by the longitudinal ac forces and found that the motion of particles can indeed be rectified, with a 
sign that depends on the details of the wall profile. Interestingly, the net current can even be obtained by applying a transverse ac drive, like a transverse ac force or transverse wall vibration [24, 25].

However, if a polarized field coupled by longitudinal force and transversal force is applied on Brownian particles, how about the competition and cooperation between them in the transport? In the present work, we study the particle transport in an asymmetrically periodic tube driven by thermal noise and polarized field by using Brownian dynamics simulation method. We emphasize on the enhancement and secondary reversal of the current by coupling the longitudinal and transverse forces together.

\section{MODEL AND METHODS}

Because most of the molecular transport occurs in the overdamped regime, the inertial effects can be neglected in the study. So the overdamped dynamics can be described by the following Langevin equations in the dimensionless form,

$$
\begin{aligned}
& \frac{d x}{d t}=f_{x}(t)+\sqrt{D} \xi_{x}(t), \\
& \frac{d y}{d t}=f_{y}(t)+\sqrt{D} \xi_{y}(t)
\end{aligned}
$$

where, $x, y$ are the two-dimensional coordinates, $\mathrm{D}$ is the diffusion coefficient, and $\xi_{x, y}(t)$ presents the Gaussian white noise with zero mean and correlation function: $<\xi_{i}(t) \xi_{j}\left(t^{\prime}\right)>=$ $2 \delta_{i, j} \delta\left(t-t^{\prime}\right)$ for $i, j=x, y .<\ldots>$ denotes an ensemble average over the distribution of noise. $\delta$ is the Dirac delta function. Two periodic forces are applied on the particles in the longitudinal and transversal directions, respectively with the forms:

$$
\begin{gathered}
f_{x}(t)=A_{x} \cos \left(\omega t+\phi_{1}\right), \\
f_{y}(t)=A_{y} \cos \left(\omega t+\phi_{2}\right),
\end{gathered}
$$

where, $A_{x}, A_{y}$ are the amplitudes of the forces in the longitudinal and transversal directions, respectively. $\phi_{1}$ and $\phi_{2}$ are the phase of the sinusoidal forces. Here, we only consider the case that the forces in the two directions have identical frequency $\omega$. The superposition of $f_{x}(t)$ and $f_{y}(t)$ gives rise to a polarized field rotating in two dimensions as shown in figure 
1. The mode of the polarized field is characterized by the phase difference $\Delta \phi=\phi_{2}-\phi_{1}$. Driven by the polarized field, the particles collide with the asymmetric tube with different forms.

FIG. 1: Polarized field by superposition of the longitudinal and transversal forces with different modes.

In this study we consider the case that the particles are confined in a tube. The asymmetric tube is sketched in Fig. 2(a) with the shape described by its half width:

$$
y(x)=a\left[\sin \left(\frac{2 \pi x}{L}\right)+\frac{\Delta}{4} \sin \left(\frac{4 \pi x}{L}\right)\right]+b,
$$

here, $a$ is the parameter that controls the slope of the tube and $\Delta$ is the asymmetric parameter of the tube shape. The parameter $b$ determines the half width at the bottleneck. $L$ is the period of the tube. The Langevin equation describing the overdamped Brownian particle can be reduced to an effective 1D Fokker-Planck equation in the absence of the longitudinal force $f_{x}[22]$ :

$$
\frac{\partial}{\partial t} P(x, t)=\frac{\partial}{\partial x}\left[D \frac{\partial}{\partial x}+V^{\prime}(x, t)\right] P(x, t),
$$

where, the effective potential

$$
V(x, t)=-D \ln \left[\frac{2 D}{f_{y}(t)} \sinh \left(\frac{f_{y}(t) y(x)}{D}\right)\right] .
$$

The transient profile of the effective potential is shown in Fig. 2 (b). The potential barriers are skewed to the right with ratchet length $L_{+}>L_{-}$. Under appropriate parameters, the maximum of the potential keeps constant, while the minimum vibrates periodically with frequency of twice of the driving frequency. This leads to periodic change on the potential 


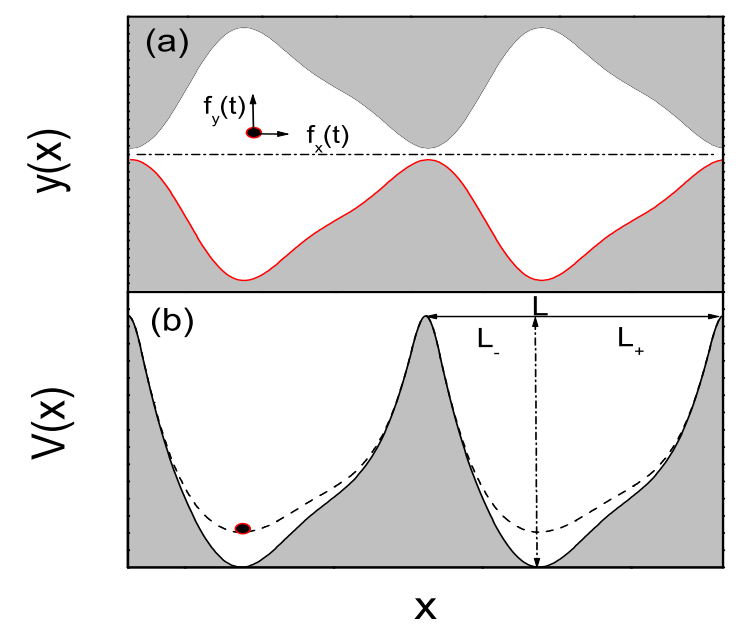

FIG. 2: Schematic of the two-dimensional tube (a) and the corresponding effective potential (b). $L=L_{+}+L_{-}$is the period of the tube, $L_{+}$and $L_{-}$indicate the asymmetry of the effective potential. The dash line and the solid line in (b) present the vibration range of the potential well.

barrier. Together with the longitudinal force, it gives rise to complex dynamics of the particle transport.

We carry out the Brownian dynamic simulations performed by integration of the overdamped Langevin equation using the standard stochastic Euler algorithm. Reflecting and periodic boundary conditions are applied in the transversal and longitudinal direction [25], respectively. The average particle velocity derived from an ensemble average of more than $10^{4}$ along the $\mathrm{x}$-direction reads:

$$
v=\langle\dot{x}\rangle=\lim _{t \rightarrow \infty} \frac{\langle x(t)\rangle}{t} .
$$

Unless otherwise noted, our simulations are under the parameters sets: $a=1 / 2 \pi, b=$ $1.2 / 2 \pi, \Delta=1.0, D=0.1, \Delta \phi=0.0$, and $L=1.0$ throughout this work.

\section{NUMERICAL RESULTS AND DISCUSSION}

We firstly study the determination of the frequency of the polarized field on the particle transport. Figure 3 presents the dependence of the mean velocity on the driving frequency for the case that longitudinal force, transversal force, and linearly polarized field are applied, 
respectively. It exhibits interesting phenomena of enlargement and secondary reversal of current originated from the polarized field as increasing the driving frequency. We find that it actually demonstrates the cooperative relationship between the longitudinal and transversal forces. In order to illustrate this, it is necessary to discuss the cases that the longitudinal and transversal forces are applied individually.

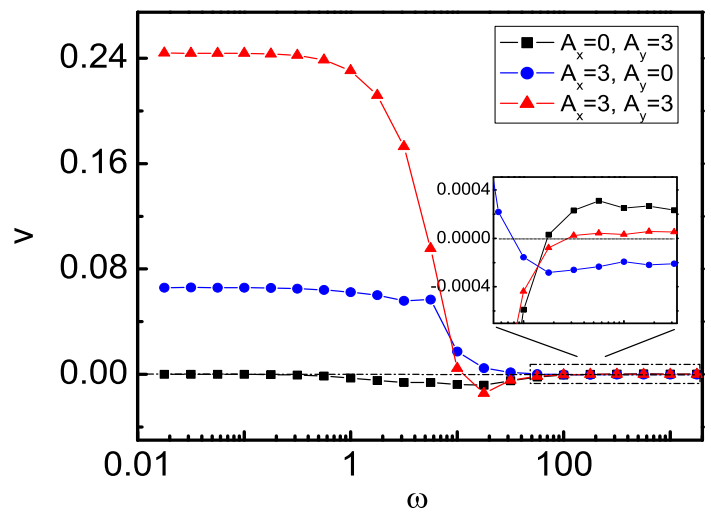

FIG. 3: (Color online) Dependence of the mean velocity on the driving frequency. The square, circle, and angle lines present the applications of transversal force, longitudinal force, and polarized field. The inset shows the mean velocity at high frequency.

In figure 3 the circle line presents the mean velocity induced only by the longitudinal force, $A_{x} \neq 0, A_{y}=0$. It can be seen that the current changes direction once as increasing the frequency. In the adiabatic limit $\omega \rightarrow 0$, the driven source can be divided into two opposite static forces $A_{x}$ and $-A_{x}$. The mean velocity can be expressed as $v=\frac{1}{2}\left[v\left(-A_{x}\right)+v\left(A_{x}\right)\right]$. In this case the particles get enough time to cross the both sides and it is easier for a particle to climb the potential well to the right than to the left, which gives rise to a positive current. As increasing the frequency, the duration which the force acts on the particles decreases in every period. The diffusion of the Brownian particles to the left bottleneck depresses the particles to climb to the right wall, which leading to a negative net current. For the extreme case $\omega \rightarrow \infty$, the particles cannot respond the applied force, which makes the current tend to be zero. So, the current changes direction from positive to negative in the absence of the transversal force.

When the Brownian particles are driven periodically only in transversal direction, $A_{x}=0$, $A_{y} \neq 0$, the behaviors of the current change dramatically as shown by the square line in 
Fig. 3. In the adiabatic limit $\omega \rightarrow 0$, the mean velocity also is $v=\frac{1}{2}\left[v\left(-A_{y}\right)+v\left(A_{y}\right)\right]$. It is obvious that no net current occurs when a transversal constant force is applied, namely, $v\left(-A_{y}\right)=v\left(A_{y}\right)=0$. Therefore, the current tends to zero in the adiabatic limit. On increasing the frequency, the Brownian particles have enough time to diffuse in the whole area. It has higher probability to collide with the right wall because the area of the right wall is larger than that of the left wall, which gives rise to a negative current. When the frequency is higher, the particles have no enough time to reach the whole area of the right wall. The probability of the particles colliding with the right wall is smaller than that with the left wall, leading to a positive current. For the extreme case $\omega \rightarrow \infty$, the Brownian particles cannot respond the driven force, which results in a zero current. So, the current changes direction once from negative to positive in the absence of the longitudinal force.

However, if a polarized field is applied, novel phenomena appear as indicated by the angle line in Fig. 3. One of the important results is that the current is enlarged remarkably when the driving frequency is not too high. For the positive current, the magnified effect becomes more evident. The current induced by a polarized field is not a simple algebraic sum of those from longitudinal and transversal forces. The amplification factor can be control by adjusting parameters such as the driving amplitude, their ratio $A_{x} / A_{y}$, the period of the tube and so on. It shows that the coupling between the longitudinal and transversal forces can improve the rectification effect of ratchets. This is very significant in applications such as particle separation. The other important result is the appearance of the secondary reversal of the current as increasing the driving frequency. As stated above, the current induced individually by longitudinal or transversal force varies direction only once upon increasing the frequency. However, by coupling the longitudinal and transversal forces together, the current can undergo reversal twice. Although the magnitude of the current in Fig. 3 is small at high frequencies (it is still within the numerical accuracy of the simulations, see Ref.[25]), as will be shown later, one can improve the current by adjusting the control parameters. So, a polarized field coupled by longitudinal and transversal forces provides a more efficient way to control the particle transport, especially to realize the enlargement of the current.

As a characteristic parameter of a polarized field, the phase difference $\Delta \phi$ affects the particle transport as well. We find that the current varies sinusoidally with a period of $\pi$ instead of $2 \pi$ as changing the phase difference (not shown). This is because that the tube owns mirror symmetry along the longitudinal axis. So, it is identical for the levorotatory 
and dextrorotatory polarized fields when acting on the particles. When $\Delta \phi=0$, $\pi$, i.e. the longitudinal and transversal forces act on the particles with the maximum amplitude synchronously, the current reaches maximum. However, if the longitudinal force is out of phase with the transversal force the current will decrease. For the cases of circular polarizations, $\Delta \phi=\frac{\pi}{2}, \frac{3 \pi}{2}$, the current is minimum.

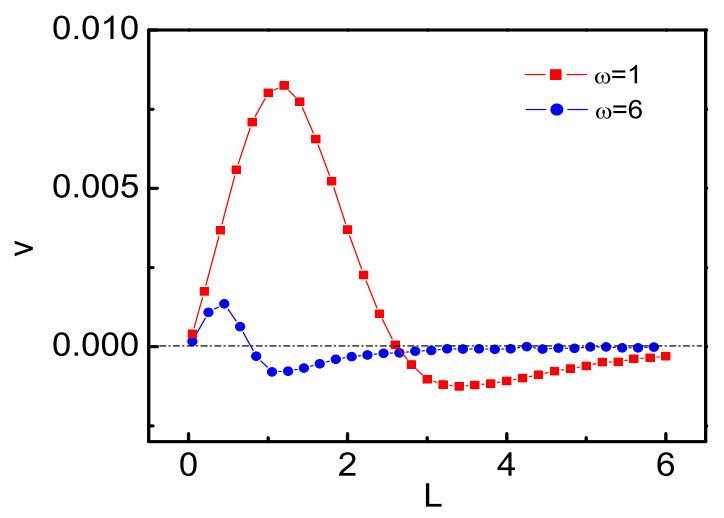

FIG. 4: (Color online) Dependence of the mean velocity on the period of the tube at frequencies $\omega=1$ and $\omega=6$. The width of the tube keeps constant. The amplitudes are $A_{x}=A_{y}=0.5$.

There exists competitive and cooperative relationships between the longitudinal and transversal forces on controlling the particle transport. Figure 4 presents the dependence of the mean velocity on the period of the tube. When the period of the tube is very small compared with the width of the tube, the aspect ratio of the tube $\max [y(x)] / L$ is large. The particle has no enough time to collide with the wall before it climbs the entropic barrier from the right wall. In this case the longitudinal force predominates in controlling the Brownian particle, which leading to a positive current. On the contrary, if the period becomes large, the aspect ratio is small. The particle will collide well with the right wall before it climbs the entropic barrier from this side, which gives rise to a negative current. Under appropriate value of period (the cross points on the longitudinal axis), the actions of the longitudinal and transversal forces reach balance, and the particle couldn't escape from the well leading the net current to be zero. For the two extreme cases, $L \rightarrow 0, \infty$, the tube tends to be straight and loses the symmetry-breaking. So, the current disappears. This gives us an insight that by adjusting the period of the system, for example stretching or compressing the tube, one can control the direction of the current. 

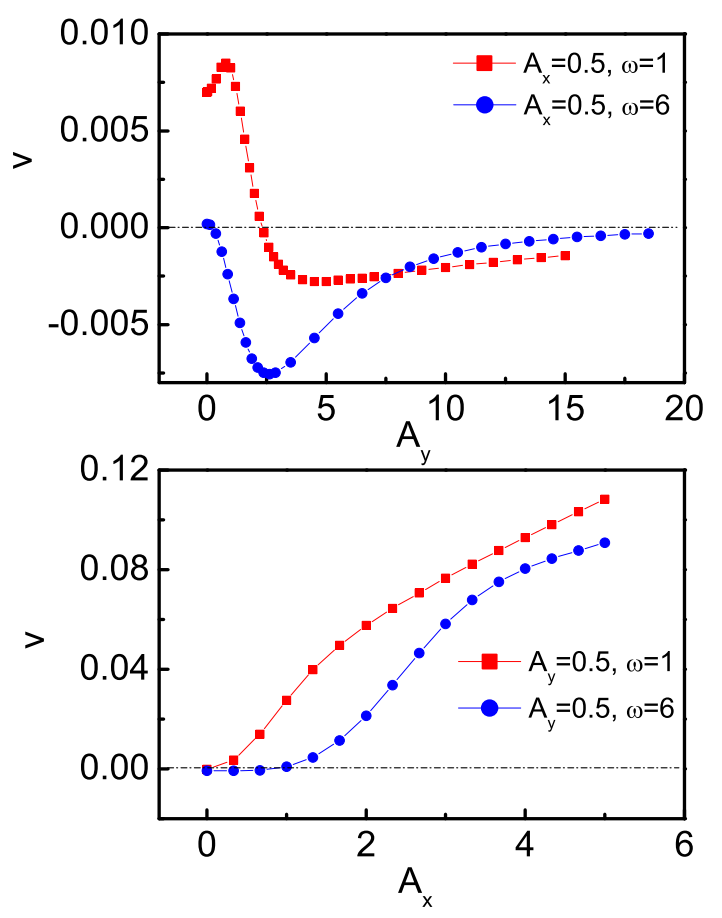

FIG. 5: (Color online) Dependence of the mean velocity on the ratio of driving amplitudes at frequencies $\omega=1$ and $\omega=6$. (a) [(b)] presents the variation of the mean velocity as increasing the transversal (longitudinal) force with fixed longitudinal (transversal) force $A_{x}=0.5\left(A_{y}=0.5\right)$.

Figure 5 shows the dependence of the mean velocity on the amplitude of $A_{y}$ and $A_{x}$, respectively. In Fig. 5 (a) [(b)], the longitudinal (transversal) force is kept constant amplitude $A_{x}=0.5\left(A_{y}=0.5\right)$ while increasing the amplitude of the transversal (longitudinal) force. At the origin of the longitudinal axis in Fig. 5, the current doesn't equal to zero, which is induced by the longitudinal and transversal forces applied individually. From figure 5 we can find that, on one hand, all the curves cross the longitudinal axis once, which indicates the competition between the longitudinal and transversal forces. The points of intersection present the balance between the longitudinal and transversal actions leading to zero current. So, by applying an addition transversal driving force, one can further control the current direction beside its enhancement function. On the other hand, there exist optimization values of the amplitude at where the current reaches absolute maxima. This illustrates the cooperative relationship between the longitudinal and transversal forces. In Fig. 5 (b), there should be a maximum if the longitudinal amplitude is strong enough. 


\section{CONCLUDING REMARKS}

In conclusion, we have studied the transport of overdamped Brownian particles confined in an asymmetrically periodic tube applying a polarized field. By coupling the longitudinal and transversal forces together, the current exhibits two types of novel phenomena: enhancement and secondary reversal. The enhanced efficiency and the current reversal can be controlled flexibly by adjusting the system parameters such as the driving amplitudes, their ratio $A_{x} / A_{y}$, the phase different, and the period of the tube. The results we have presented may have a potential application in many processes, such as particle separation, diffusion in biological membrane, transport in zeolites, controlled drug releases, and diffusion in manmade materials.

Clearly, the model is too simple to provide a realistic description of real systems. However, the results we have presented have wide applications in may processes, such as the vortex ratchet in superconductor [12 15, 17], gating ratchet with cold atoms in optical lattice [18], diffusion of ions and macromolecular solutes through the channels in biological membranes [26], transport in zeolites [27], and diffusion in man-made periodic porous materials [21].

\section{ACKNOWLEDGMENTS}

This work was supported in part by National Natural Science Foundation of China with Grant No. 30600122, 10947166 and GuangDong Provincial Natural Science Foundation with Grant No. 06025073. Y. F. He also acknowledges the Research Foundation of Education Bureau of Hebei Province (Grant No. 2009108).

[1] F. Julicher, A. Adjari, and J. Prost, Rev. Mod. Phys. 69, 1269(1997).

[2] P. Hänggi and F. Marchesoni, Rev. Mod. Phys. 81, 387(2009).

[3] P. Reimann, C. Van den Broeck, H. Linke, P. Hänggi, J. M. Rubi, and A. Perez-Madrid, Phys. Rev. Lett. 87, 010602(2001).

[4] J. Rousselet, L. Salome, A. Adjari, and J. Prost, Nature 370, 446(1994).

[5] L. P. Faucheux, L. S. Bourdieu, P. D. Kaplan, and A. J. Libchaber, Phys. Rev. Lett. 74, 1504(1995). 
[6] I. Derenyi, C. Lee, and A. L. Barabasi, Phys. Rev. Lett. 80, 1473(1998).

[7] C. S. Lee, B. Janko, I. Derenyi, and A. L. Barabasi, Nature 400, 337(1999).

[8] M. O. Magnasco, Phys. Rev. Lett. 71, 1477(1993).

[9] P. Reimann, Phys. Rep. 290, 149(1997); J. D. Bao and Y. Z. Zhuo, Phys. Lett. A 239, 228(1998); B. Q. Ai, L. Q. Wang, and L. G. Liu, Chaos, Solitons Fractals 34, 1265(2007).

[10] P. Reimann, R. Bartussek, R. Haussler, and P. Hänggi, Phys. Lett. A 215, 26(1996).

[11] C. R. Doering, W. Horsthemke, and J. Riordan, Phys. Rev. Lett. 72, 2984 (1994).

[12] S. Savel'ev and F. Nori, Nature materials 1, 179(2002); S. Ooi, S. Savel'ev, M. B. Gaifullin, T. Mochiku, K. Hirata, and F. Nori, Phys. Rev. Lett 99, 207003(2007); S. J. Bending, D. Cole, S. Savel'ev, F. Nori, and T. Tamegai, Physica C 460-462, 1266(2007).

[13] D. Cole, S. Bending, S. Savel'ev, A. Grigorenko, T. Tamegai, and F. Nori, Nature materials $5,305(2006)$.

[14] J. E. Villegas, S. Savel'ev, F. Nori, E. M. Gonzalez, J. V. Anguita, R. García, and J. L. Vicent, Science 302, 1188(2003).

[15] Clécio C. de Souza Silva, Joris Van de Vondel, Mathieu Morelle, and Victor V. Moshchalkov, Nature 440, 651 (2006).

[16] S. Savel'ev, F. Marchesoni, P. Hänggi, and F. Nori, Phys. Rev. E 70, 066109(2004).

[17] E. Kalman, K. Healy, and Z. S. Siwy, Europhys. Lett. 78, 28002(2007).

[18] R. Gommers, V. Lebedev, M. Brown, and F. Renzoni, Phys. Rev. Lett. 100, 040603 (2008); P. H. Jones, M. Goonasekera, and F. Renzoni, Phys. Rev. Lett. 93, 073904 (2004); R. Gommers, S. Denisov, and F. Renzoni, Phys. Rev. Lett. 96, 240604 (2006).

[19] M. Bier, M. Kostur, I. Derényi, and R. D. Astumian, Phys. Rev. E 61, 7184(2000).

[20] M. Kostur and L. Schimansky-Geier, Phys. Lett. A 265, 337(2000).

[21] T. Chou and D. Lohse, Phys. Rev. Lett. 82, 3552(1999); L. Liu, P. Li, and S. A. Asher, Nature 397, 141(1999); S. Matthias and F. Muller, Nature 424, 53(2003).

[22] D. Reguera and J. M. Rubi, Phys. Rev. E 64, 061106(2001); D. Reguera, G. Schmid, P. S. Burada, J. M. Rubi, P. Reimann, and P. Hänggi, Phys. Rev.Lett. 96, 130603(2006); P. S. Burada, G. Schmid, P. Talkner, P. Hänggi, D. Reguera, and J. M. Rubi, BioSystems 93, 16(2008)

[23] B. Q. Ai and L. G. Liu, Phys. Rev. E 74, 051114(2006); B. Q. Ai, Phys. Rev. E 80, 011113(2009). 
[24] F. Marchesoni and S. Savel'ev, Phys. Rev. E 80, 011120(2009).

[25] B. Q. Ai, J. Chem. Phys. 131, 054111(2009).

[26] B. Hille, Ion Channels in Excitable Membranes (Sinauer Associates, Sunderland, MA, 2001).

[27] J. Karger and D. M. Ruthven, Diffusion in Zeolites and Other Microporous Solids (Wiley, New York, 1992). 